\title{
TrsI_SSC3.Up >
}

Hb1.8 TGCCAGCCTACAACCACAGCCACAGCAACTCAGGATCCGAGCCGCGTCTGCGACCtgecggcaaagtctccctgtatctggctggtacccctgggggcattccctatccccggtccagaatgtgtct

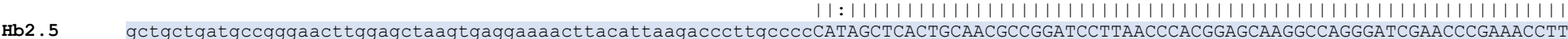

Hb1.8 gtcctcagcccctcctcttccctacctgtcacagccetctctgcttccaacagtgctcagccgagtgtgggacgggaatccagcgacgctctgtggtctgccttgggagt

SSC3 TGGTTCCTAGTCCTTAACCACGGAGGCATGAAGGGAACTCCTGGATGTAAGTTTTCAT-TTCCTTGGGAACAATGGCCAAGAGCTCAGTTGCCAGCTCACATGGTAATTGCACATTTCGTATGTAAG

Hb2.5 $11111111111111111111111111111111111111111111111111111111: 1111111111111$

$<$ TrSI_SSC4.Dn

(b) $\quad \mathrm{Hb} 1.8$

$\operatorname{SSC} 4$

$\mathrm{Hb} 2.5$

$\mathrm{Hb} 1.8$

$\operatorname{SSC} 4$

$\mathrm{Hb} 2.5$

$\operatorname{SSC} 4$

$\mathrm{Hb} 2.5$

Hb1.8 GTCCTCAGCCCCCTCCTCTTCCCCTACCTGTCACAGCCCTCTCTGCTTCCCAACAGTGCTCAGCCGAGTGTGGGACGGGAATCCAGCGACGCTCTGTGGTCTGCCTTGGGAGT

TrSI_SSC4.Up>

\section{c}

GGCCCCACACCCCCTCAGCCAAGTCCCCATCCCTGGTGCCCCACATCCACCACACCCTCTCAGAGCCTCTCCAACTCGGTTAAATAAACTTTTATTGAGCAAGCACCTGCTGTATGCTAGATGCTGC

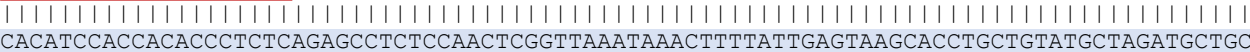

1.8 tgccagcctacaaccacagccacagcaactcaggatccgagccgcgtctgcgaccTGCCGGCAAAGTCTCCCTGTATCTGGCTGGTACCCCTGGGGGCATTCCCTATCCCCGGTCCAGAATGTGTCT TGATGCCGGGAACTTGGAGCTAAGTGAGGAAAACTTACATTAAGACCCTTGCCCCTGCCGGCAAAGTCTCCCTGTATCTGGCTGGTACCCCTGGGGGCATTCCCTATCCCCGGTCCAGAATGTGTCT TGATGCCGGGAACTTGGAGCTAAGTGAGGAAAACTTACATTAAGACCCTTGCCCCatagctcactgcaacgecggatccttaacccacggagcaaggccagggatcgaacccgaaaccttatggtt GTCCTCAGCCCCTCCTCTTCCCCTACCTGTCACAGCCCTCTCTGCTTCCCAACAGTGCTCAGCCGAGTGTGGGACGGGAATCCAGCGACGCICTGTGGTCTGCCTTGGGAGIGGGGAGGCCCATGG $<$ TrSI_SSC3.Dn 